\title{
Feasibility Analysis on the Transaction Integration of Air Pollution Emission in 'Dianzhong' Economic Zone
}

\author{
Wen Gao \\ China, Oxbridge College, Kunming University of Science and Technology, China \\ panpanbear@hotmail.com
}

Keywords: "Dianzhong” economic zone; the transaction of air pollution emission

\begin{abstract}
Dianzhong” economic zone consists of Kunming city, Qujin city, Yuxi city and Chuxiong City. The position of the economic zone is central of Yunnan province. In "13th FiveYear Plan", the "Dianzhong" economic zone is considered the core area, the power and the leader of the economic development of Yunnan Province. The population accounts for $1 / 3$ of the Province, the gross domestic product accounts for $1 / 2$ of the Province, the total industrial and agricultural output value accounts for $2 / 3$, the total industrial output value accounts for $4 / 5$, and the grain output accounts for $1 / 3$. But in this zone, there are more than $60 \%$ industries firms with air pollutions effecting the living conditions and human health. It is necessary to establish the transaction of air pollution emission to control the total output of air pollution taking reference from America and Europe. The paper will describe feasibility of the integration of the transaction of air pollution emission in "Dianzhong" zone firstly, on this basis, the idea to set up the market of air pollution emission transaction is put forward.
\end{abstract}

\section{The General Situation of “Dianzhong” Economic Zone}

\subsection{The process of development in Dianzhong” economic zone.}

From 2009 to 2010. During this period, the construction of the economic circle in “Dianzhong” economic zone was fully started, and the administrative divisions between cities were restrained.

The comprehensive infrastructure framework system to resolve, co-ordinate, build and share has begun to take shape, and the economic circle has begun to show its influence and impetus on the surrounding regional economy.

From 2011 to 2015. At the appointed time, the expressway, high speed railway and intercity rail transit network in the main cities in "Dianzhong" are basically completed. The construction of the economic circle in economic zone is advancing in depth. The construction of resources saving, environmental friendly and industrial optimization has achieved remarkable results, and the industrial structure and growth side of resource conservation, ecological civilization and environment friendly the pattern and consumption pattern were initially formed. The population in the economic circle reached 20 million 900 thousand people, GDP exceeded 868 billion yuan, and GDP per capita was close to 42 thousand yuan, and various eco-environmental indicators began to improve.

From 2016 to 2020. At this stage, the main tasks of comprehensive supporting construction of resources saving, environmental friendly and industrial optimization in the economic circle of central Yunnan are basically completed. The system mechanism of resource conservation, environment friendly and ecological civilization is basically formed, and the new industrialization and urbanization development model is basically established as the Yungui Plateau of the national ecological barrier. The pressure of ecological protection will be greatly alleviated, and the pattern of coordinated economic and social development with population, resources and environment will basically take shape. 


\subsection{Strategic positioning of "Dianzhong“economic zone}

Kunming, Qujing, Yuxi and Chuxiong have four cities in "Dianzhong” Economic Zone. Around 2010, the region concentrated about $40 \%$ of the population of Yunnan and $60 \%$ of the total economic total. It is the area of large and medium-sized cities in Yunnan, the convenience of transportation and logistics, the solid industrial base, the comprehensive economic strength and the relatively developed undertakings of science and education and education. The four cities in "Dianzhong“ economic zone should speed up the construction of a modern industrial system with complementary advantages, progressive layers, reasonable layout and distinctive features.

Taking Kunming as the center and taking Qujing, Yuxi and Chuxiong as the support, the spatial development pattern of the central Yunnan urban economic circle is integrated with the main traffic axis as the link.

To strengthen Kunming's scientific and technological innovation, trade and circulation, information, tourism, culture and comprehensive service functions, the construction of regional international transportation hub, trade logistics center, historical and cultural city, landscape garden city. Qujing, Yuxi and Chuxiong and other node cities should rely on the characteristics of resources and comparative advantages to strengthen the industrial division of labor and cooperation and docking, to achieve complementary advantages and dislocation development, and to form a city with distinctive national characteristics and distinctive industrial characteristics.

To improving the international transport corridor and strengthening the land hub functions for Southeast Asia and South Asia. We should strengthen the infrastructure construction of intercity rapid rail transit and communication and enhance the level of regional integration.

To building the production base of high quality and characteristic agricultural products, developing the processing industry of agricultural products, steadily improving the quality and efficiency of agricultural products, and promoting the agricultural cooperation with the surrounding countries.

To strengthen the control of plateau lakes and the prevention and control of soil erosion on the plateau in Dianchi, the ecological pattern of plateau lakes as the main body, woodland, water surface, banded surround and block phase are constructed.

The key industrial clusters in economic zone are as follows: "two smoke" and supporting industrial clusters mainly in Yuxi, Kunming, Qujing and Chuxiong; the high-tech industrial clusters of Kunming, Qujing and Yuxi; the coal chemical industry cluster in Qujing; the industrial clusters of Kunming, Qujing and Yuxi, with Kunming and Chu. Male based biopharmaceutical industry; Kunming and Chuxiong's cultural industries; Kunming, Yuxi, Chuxiong and Qujing tourism industries.We should form a "huan Dian" industrial intensive area, "Qu Zhan Ma" industrial intensive areas, "Hongta district - Jiangchuan - Tonghai" industrial intensive areas, "Nanhua Chuxiong" industrial intensive areas, "Anning Lufeng" industrial intensive areas, "Xuanwei Fuyuan" industrial intensive areas, "Luliang - Shizong - Luoping" industrial intensive areas, "E Shan" Xinping Yuanjiang "industrial intensive area" and other 8 industrial intensive areas.

\section{Feasibility of the Integration of the Transaction of Air Pollution Emission in "Dianzhong" Economic Zone}

2.1 The overall level of ecological environment in "Dianzhong” Economic Zone has been maintained well, and the forest coverage rate is over 50\%. Large reserves of mineral resources and high economic value have concentrated most of the mineral resources such as phosphorus, iron, lead and coal in the province. The economic circle of "Dianzhong" is the fastest growing area in Yunnan Province. Especially since the implementation of the strategy of the western development, the economic and social development has made remarkable achievements and has entered the best period of development in history. In 2012, the economy of the central Yunnan city $44.06 \%$ of the population in the province and $29 \%$ of the territory of the province realized $65.56 \%$ of the total GDP of the province, $66.17 \%$ of the total local fiscal revenue, $67.54 \%$ of the total retail sales of social consumer goods and $56.19 \%$ of the investment in fixed assets in the whole society. $80.49 \%$ of 
total imports and exports and 82.63\% of industrial added value. In 2015, Yunnan's gross domestic product (GDP) was 1 trillion and 371 billion 788 million; the per capita GDP of the province was 29100.91 yuan, which was lower than the national average. From all levels, the total GDP in Kunming, Qujing and Yuxi is 397 billion, 163 billion 30 million and 124 billion 570 million respectively. From the per capita GDP, Kunming, Yuxi and Diqing state ranked the top three, the per capita GDP of Kunming was 59915.48 yuan, 9619.72 US dollars, Yuxi per capita GDP was 8507.15 US dollars, Chuxiong per capita GDP was 4490.59 US dollars fifth, Qujing per capita GDP was sixth to 2. By the end of 017, the total GDP of 5 cities in Kunming, Qujing, Honghe, Yuxi and Dali exceeded 100 billion yuan.

The urban economic circle of "Dianzhong” economic zone has gathered 78\% colleges and 67\% scientific research institutes, 85\% engineering technology research centers, 94\% key laboratories, 90\% high-tech enterprises, 95\% technical innovation talents, and 100\% AAA hospitals in the province. The city of central Kunming, which is the core of the city, is growing steadily, and its ability to gather and absorb the population has been increasing. The urban network system of the urban economic circle in "Dianzhong" economic zone is basically formed, and the conditions for building the urban agglomeration are already available, which can provide support for the establishment and rapid development of regional industrialization, the emission trading of air pollutants.

\subsection{A certain legal basis}

Since 1980s, the national and local environmental protection departments have promulgated a series of laws and regulations regulating the emission trading, which laid a certain legal basis for the regional emission trading. 1988 the State Environmental Protection Administration promulgated the "Interim Measures" water pollutant discharge permit management, which stipulates that "local sewage units can mutually adjust emission index of water pollutants, and to determine the Beijing, Shanghai, Tianjin and other 18 city water pollutant discharge permit pilot work. In 1994, the former State Environmental Protection Agency launched a pollutant discharge permit system throughout the country. In 1996, the State Council approved the national environmental protection "Jiuwu” plan, the 2010 vision target and the total emission control plan for the national major pollutants during the period of "Jiuwu" and identified 12 kinds of pollutants to be controlled by the total amount. The total amount control was first formally entered in China's pollution control policy system and was pushed throughout the country. In June 10, 1997, the State Environmental Protection Administration also issued the "total emission control plan for major pollutants in the country" during the "95" period. Total control has officially entered China's pollution control policy system. In September 2000, the third revised version of the law on air pollution control began to be implemented. For the first time, the legal status of total emission control and emission permit was clarified. The State Environmental Protection Administration (State Environmental Protection Administration) implemented the regulations on the management of pollutant discharge license (Draft) on 14 July 2004 and prepared the policy for the full implementation of the pollutant discharge permit system.

In addition to the legal system at the national level, many local governments have also formulated the local trading system for emission permits. The Kunming environmental energy exchange, which was set up in August 2008. The main objects of exchange transactions are emission rights and emission rights. For example, chemical oxygen demand and sulfur dioxide desulphurization in the thermal power industry will be traded on the exchange in Dianchi. The exchange is the entire southwest China, which is the first pollutant discharge exchange. Emissions trading is ahead of other provinces in the southwest. In February 2016, the Yunnan provincial Environmental Protection Department collected opinions on the guidance of the Ministry of environmental protection on strengthening the work of local environmental protection standards. 2017, Honghe Environmental Protection give the document of silver Shuo Mining Co. is not the normal operation of air pollution control facilities, air pollutant emissions exceed the standard implementation of punishing illegal acts, a daily penalty of a fine of 23 million yuan. This is the largest penalty in Yunnan so far. It also shows the determination and strength of the Yunnan 
provincial government in the prevention and control of air pollutant emissions.

\subsection{The successful experience of emission trading at domestic and abroad.}

The earliest practice of emissions trading originated in the United States and was fully promoted in 1990s and achieved great success. In the 1990 2006, the power generation in the United States has increased by $37 \%$ and the consumption of coal has greatly increased, the total amount of SO2 emission decreased by $40 \%$, and the NO2 emission decreased by $48 \%$. The emission of other major pollutants has also been greatly reduced, making the level of wet sulphate deposition down by $25 \%$ $\sim 40 \%$ in most of the central and western parts of the United States than in 1990. Subsequently, western countries such as Australia, Canada and Germany also made positive and effective explorations on emissions trading system and achieved certain results.

The right to discharge air pollutants in China was launched in 1991 in 16 cities such as Shanghai and Tianjin. In 1994, 6 pilot cities in Shantou, Kaiyuan, Liuzhou, Taiyuan, Pingdingshan and Guiyang carried out pilot tests on air pollution permits trading and gained initial experience. During the period of "fifteen", the focus of environmental protection work in China shifted to the total amount of pollutant emission control. Before and after 2001, a number of pilot projects were carried out throughout the country, such as the pilot project of the SO2 emission trading of the Asian Development Bank in Taiyuan, and the pollution emission trading projects of the United States Environmental Association in Nantong, etc. Under the impetus of these projects, a number of emission trading cases have been completed and accumulated rich experience. In 2002, 7 provinces and cities in East, Shanxi, Jiangsu, Henan, Shanghai, Tianjin and Liuzhou launched pilot projects for total sulfur dioxide emission control and emission trading. In 2003, Jiangsu Taicang port environmental protection power generation Co., Ltd. and Nanjing Xiaguan Power Plant reached SO2 emissions trading in different places, creating a precedent for China's trans regional transactions. The pilot of water pollutant emission trading was also carried out in this stage. In November 10, 2007, the first emission trading center in China was set up in Jiaxing, Zhejiang, which marks the normalization and institutionalization of the emission trading in China. The Kunming environmental energy exchange, which was set up in August 2008. The main objects of exchange transactions are emission rights and emission rights. Among them, the right to discharge includes the chemical oxygen demand in the process of governance in Dianchi, sulfur dioxide in the thermal power industry and so on. The exchange is the entire southwest China, which is the first pollutant discharge exchange. The first practice of the emissions trading of air pollutants at home and abroad provides valuable experience for the implementation of the emissions trading of air pollutants in the new area of Yunnan Province.

\section{Conclusion}

Based on the above analysis, we should make a conclusion that it is feseability to establish the regional air pollutant emissions trading system in the 'Dianzhong' Economic Zone (Kunming, Yuxi, Chuxiong and Qujing). Through the establishment of regional integration of trade to protect environmental resources, maintain the sustainable development of the region and make up for the deficiencies of the existing sewage charges system.

\section{References}

[1] The report of the situations of environment In Yunnan, Yunnan environment protection department, 2017.

[2] The report of the situations of environment In Yunnan, Yunnan environment protection department, 2016.

[3] The report of the situations of environment In Yunnan, Yunnan environment protection department, 2015.

[4] The report of the situations of environment In Yunnan, Yunnan environment protection 
department, 2014.

[5] Jinshu Ma, Improve the development in the 'Dianzhong' economic zone, Yunnan daily, September 12, 2012.

[6] Junli Zhang, The situations and the plan of environment in the 'Dianzhong' economic zone, Environment and science, vol.23, 2013.

[7] The plan of the development about the 'Dianzhong' economic zone (2014-2024) , General Office of Yunnan provincial government, 2014.

[8] Minde Leng, improve the development between Kunming and Yuxi based on 'Dianzhong' economic zone, Yuxi daily, July 2011.

[9] Dele Niu, studies on the economic development in 'Dianzhong' economic zone, the journal of northwest normal university, vol. 4, 2013. 